\title{
Single visit endodontics resulted in less pain than multiple visits
}

Albashaireh ZSM, Alnegrish AS. Postobturation pain after single- and multiple-visit endodontic therapy. A prospective study. J Dentistry 1998; 26: 227-232

Objective To determine whether there is any significant difference in the incidence of post-obturation pain after single-and multiplevisit root canal treatment.

Design A randomised controlled trial in a hospital setting.

Intervention Three hundred consecutive patients receiving root canal treatment were assigned randomly into either single- or multiplevisit groups. The canals of all teeth were prepared and filled by a single operator using the step-back and lateral condensation techniques.

Outcome measures The frequency of post-obturation pain was recorded and evaluated over 30 days. The data were analysed statistically to determine the relationship, if any, between the pain experienced and pulpal vitality, tooth type, pre-operative pain, and the sex and age of the patient.
Results Nine patients were excluded, as they failed to attend for reviews. A significantly higher incidence $(\mathrm{P}<0.01)$ of post-obturation pain was found in the multiple-visit group (38\%) than in the single-visit group $(27 \%)$ within 24 h of obturation. No significant correlation was found between post-obturation pain and any other factor, with the exception that teeth which had non-vital pulp before treatment were associated with a significantly greater $(\mathrm{P}<0.005)$ incidence of postobturation pain.

Conclusions Pain was significantly higher in the multiple-visit RCT group and significantly associated with the treatment of the non-vital pulp.

Address for reprints: Dr Z Albashaireh, Faculty of Dentistry, Jordan University of Science and Technology, Irbid, Jordan.

\section{Commentary}

One of the problems with root canal treatment is the incidence of postobturation pain, which, although often short-lived, is embarrassing for the dentist and annoying for the patient, especially if the tooth was symptomless before the start of treatment. Pain may arise after root canal treatment, because of instrumentation beyond the apical constriction and periradicular extrusion of debris, micro-organisms, irrigant and root filling materials, resulting in acute inflammatory response and subsequent pain. Preparing the root canal from the coronal aspect before apical preparation, helps reduce the likelihood of extrusion of debris and micro-organisms.

All the teeth in this study involved step-back preparation but the authors note that the results may have been different if a step-down or initial coronal flaring had been undertaken.

Three hundred patients were treated with 150 in each group. In the multiple visit group it is not clear how many appointments were required or how long after preparation the teeth were obturated. Only nine patients were lost from the study, with three patients eliminated because surgery was required 2 weeks after obturation. It would be interesting to know whether pain was one of the reasons for surgery.

Both groups experienced pain more frequently in the first $24 \mathrm{~h}$; no patient had pain after 30 days. Overall, more pain was associated with multiple-visit treatment with non-vital teeth giving more pain than vital cases. The authors are unable to provide compelling reasons why this should be so other than microbial leakage through a temporary restoration and repeated instrumentation. However, no intracanal medicament was used between visits. Biomechanical preparation and irrigation of the root canal does not remove all the microbial flora from the root canal system and an intracanal medicament is necessary to provide disinfection, before obturation ${ }^{1}$. Micro-organisms remaining in the root canal system could multiply to high levels and this may have contributed to the pain experienced in this group. More details on pre-operative pain experience would have been helpful when analysing the relationship between pre-operative symptoms and post-operative pain.

Finally, although pain is an important issue, it is not the only factor determining ultimate success in root canal treatment. It is known that success of non-vital root canal treatment cases is affected adversely by completing them in a single visit ${ }^{2}$ and long-term followup of these patients would be of interest. This study confirms that the treatment of vital cases, without acute periradicular periodontitis, in a single visit is a reasonable treatment option.

1. Sjogren $U$, Figdor $D$, Spangberg $L$, Sundqvist $G$. The antimicrobial effect of calcium hydroxide as a short-term intracanal dressing. Int Endod J 1991; 24:119-125.

2. Sjogren U, Figdor D, Persson S, Sundqvist $G$. Influence of infection at the time of root filling on the outcome of endodontic treatment of teeth with apical periodontitis. Int Endod J 1997; 30:297-306.

\section{William Saunders}

University of Dundee, Dundee, UK 